\title{
First Results from NICHE and the NICHE-TALE Hybrid Detector
}

\author{
D.R. Bergman*1, J.F. Krizmanic ${ }^{2,3,4}$, K. Nakai ${ }^{5}$, Y. Omura ${ }^{5}$, and Y. Tsunesada ${ }^{5,6}$, for \\ the Telescope Array Collaboration ${ }^{\dagger}$ \\ ${ }^{1}$ Dept. of Physics \& Astronomy and High Energy Astrophysics Inst., University of Utah, Salt \\ Lake City, UT, USA \\ ${ }^{2}$ Center for Research and Exploration in Space Science \& Technology \\ ${ }^{3}$ NASA/Goddard Space Flight Center, Greenbelt, MD, USA \\ ${ }^{4}$ University of Maryland, Baltimore County, Baltimore, MD, USA \\ ${ }^{5}$ Graduate School Science, Osaka City University, Osaka, Japan \\ ${ }^{6}$ Nambu Yoichiro Institute for Theoretical and Experimental Physics, Osaka City University, \\ Osaka, Japan \\ E-mail: bergman@physics . utah.edu
}

\begin{abstract}
The Non-Imaging CHErenkov Array (NICHE) is an array of 14 non-imaging air-Cherenkov light collectors with an inter-counter separation of $100 \mathrm{~m}$, a 3 "-PMT in each counter and a $45^{\circ}$-Winston cone defining the angular aperture. The NICHE array is situated 600-1000 $\mathrm{m}$ from, and in the view of, the TALE fluorescence detectors. NICHE and TALE can work together as a hybrid nonimaging/imaging air-Cherenkov detector for cosmic rays for energies above $1 \mathrm{PeV}$. In this talk, we will present the first results of NICHE working independently from TALE: shower directions, fits to the Cherenkov lateral distribution and the implied energy distributions; and the first results of the NICHE-TALE hybrid: confirmation of profile constrained angular fits in TALE and the dependence of the direct-Cherenkov lateral time-width distributions in NICHE on the depth of shower max as measured by TALE.
\end{abstract}

36th International Cosmic Ray Conference -ICRC2019-

July 24th - August 1st, 2019

Madison, WI, U.S.A.

\footnotetext{
* Speaker.

${ }^{\dagger}$ for collaboration list see PoS(ICRC2019)1177
} 


\section{Introduction}

The measurement of the nuclear composition of cosmic rays (CRs) in the Knee region of the $\mathrm{CR}$ spectrum, $\gtrsim 1 \mathrm{PeV}$, is made difficult due to uncertainties inherent to measurement techniques and dependence on hadronic Monte Carlo simulation models. However, it is important to make the measurement of the composition of CRs and its evolution with energy both to confirm the widely adopted Peters cycle[1] model of rigidity-dependent cutoffs of the various galactic CR nuclear components and to look for galactic and extra-galactic photons at this energy just above the highest energy photons ever detected (to look for the PeVatrons).

One way to bypass the problems with particle-based detection of EASs, is to use an entirely optical detection technique, combining imaging and non-imaging Cherenkov detectors, to observe EASs. These detectors are sensitive to the bulk of the EAS, unlike typical particle-based detectors. The Non-Imaging CHErenkov Array(NICHE) has been designed to work with the Telescope Array (TA) Low Energy extension (TALE) fluorescence telescopes in this way. The TALE telescopes work as fixed Imaging Air Cherenkov Telescope (IACTs) which can reconstruct showers using primarily Cherenkov light well outside the Cherenkov cone, typically $2^{\circ}-5^{\circ}$ away. By placing a non-imaging Cherenkov array within this field-of-view we create a hybrid Cherenkov detector.

The methods of reconstruction for NICHE itself have been presented before [2, 3]. We rely on the Cherenkov Light Distribution (CLD) in which the lateral profile of the Cherenkov light foot print is used to determine both the energy and the compositions of the shower. The measurement of the CLD shape within $120 \mathrm{~m}$ of the shower axis is necessary for determining the nuclear composition of the primary particle. We also use the Cherenkov time-Width Lateral Distribution (CWLD) which determines the time-width as function of the distance to the shower axis. It is sensitive to primary composition for distances greater than $150 \mathrm{~m}$ from the shower axis, and is thus complementary to the CLD.

\section{Current Status}

The details of construction of the current NICHE array are given in another presentation at this conference [4]. We will here highlight the data collection history of the experiment. We look at the numbers of coincidences between triggers of various multiplicities we've collected versus the date, as well as the total number of events where a Cherenkov plane front could be fit to the coincident counters. This history is shown in Figure 1. The typical counter trigger rate is $0.35 \mathrm{~Hz}$ on clear nights, while the rate of plane-fit coincidences $(M \geq 4)$ is about $0.4 \mathrm{~Hz}$.

The initial deployment of 10 counters took place in September 2017. Automated collection began with the permanent installation of the WLAN antenna in April 2018. Four mode detectors were deployed in September 2018, but problems during commissioning delayed operations of 14 counters until February 2019.

We fit the events with three or more counters in coincidence to the assumption that the Cherenkov wavefront was a plane. The observed showers cover zenith angles out to $45^{\circ}$ as expected. The distributions of plane normals is shown in Figure 2.

We have also measured the background light level by taking a series forced triggers each of counter with the shutter closed and the HV on, and then with the shutter open. Taking the 


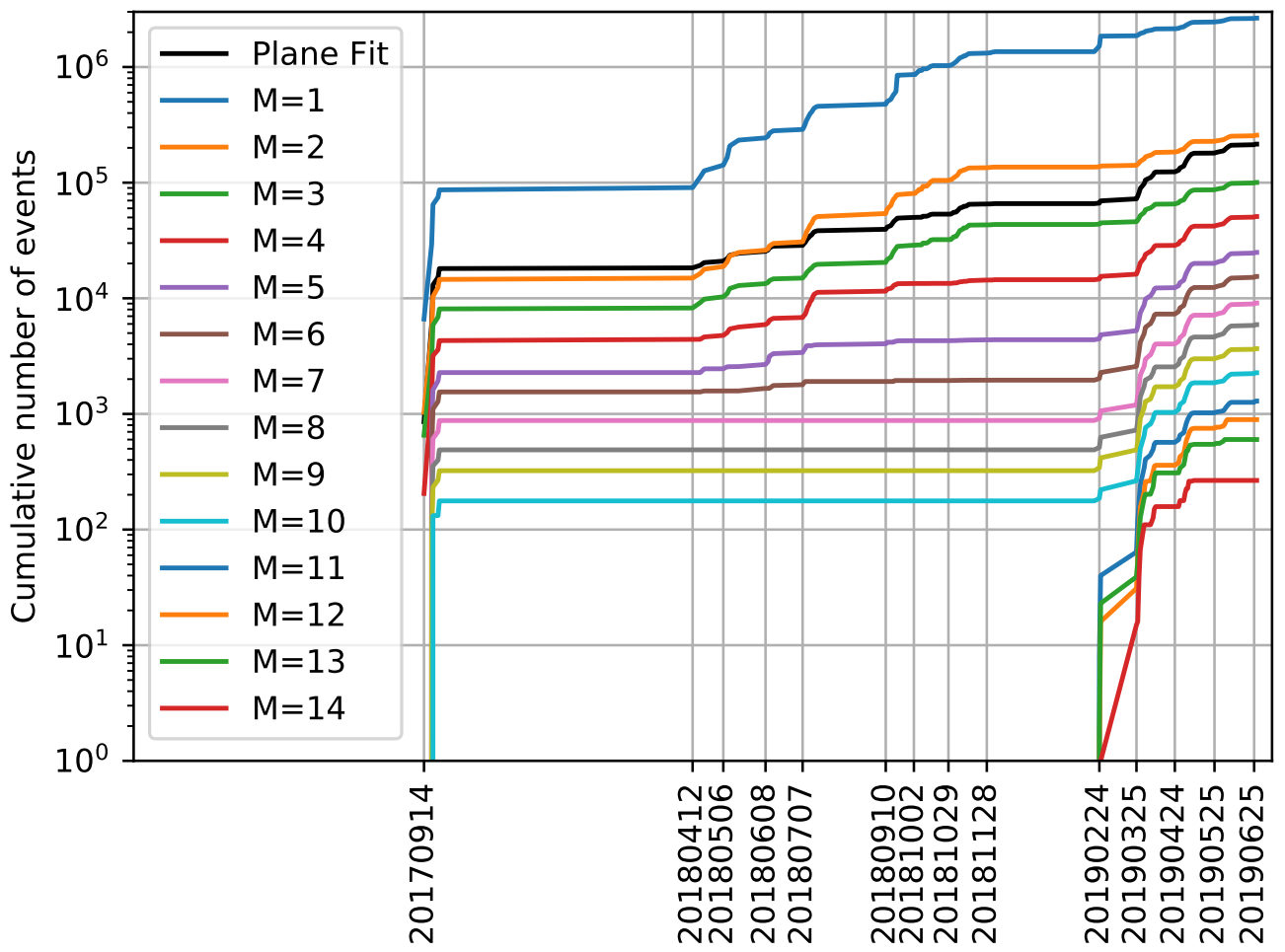

Figure 1: The NICHE data collection history by day. The exclusive number of multiplets of data by day. The number of events that could be fit to a plane by day.
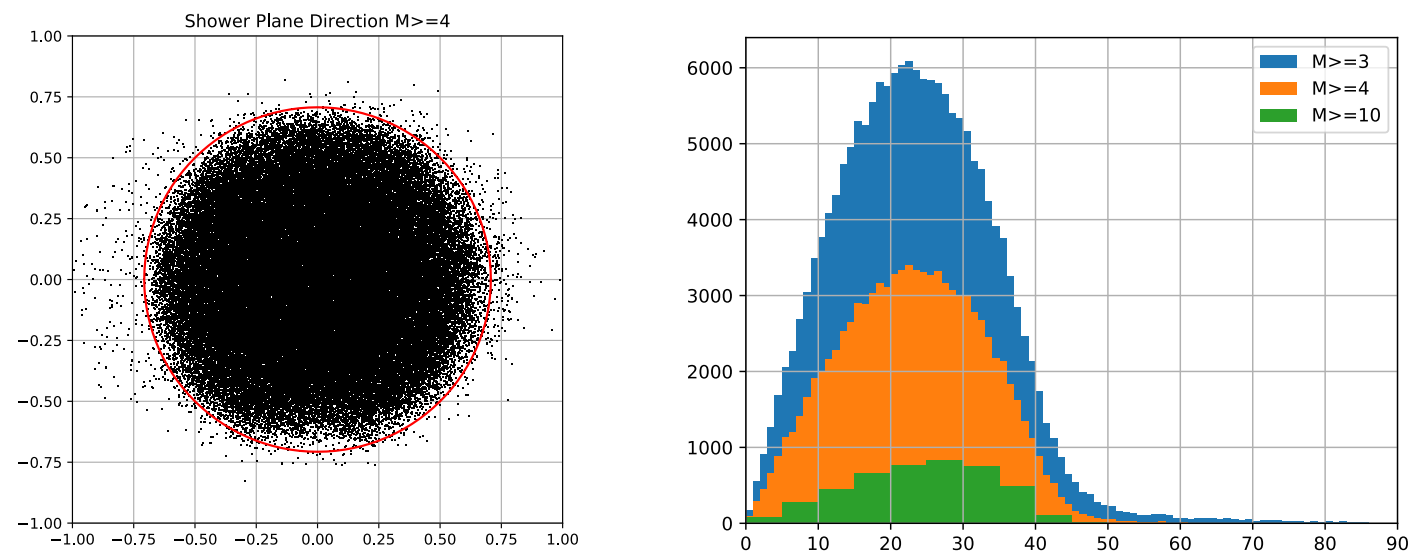

Figure 2: Left: The projection of shower front plane normal vectors onto the $x y$-plane for events with 4 or more counters. The circle containing zenith angles out to $45^{\circ}$ is shown. Right: The distributions of zenith angles for multiplicities of 3 or more, 4 or more and 10 or more counters in coincidence. 
FFT of each of these triggers, averaging the power over all the shots and subtracting the closed from the open in quadrature, we find the additional noise from the sky. An example is shown in Figure 3. The night shown was a clear night. We can reproduce the FFT with a background rate of 71.9 photoelectrons/ns on a PMT with a risetime, $r$, of $2.5 \mathrm{~ns}$ and a falltime, $f$, of $10.7 \mathrm{~ns}$ in the normalized response function[5]

$$
I(t)= \begin{cases}C_{0} \exp \left(\left(\frac{t}{r}\right)^{(2-t / 2 r)}\right) & t<0 \\ C_{0} \exp \left(-\left(\frac{t}{f}\right)^{\max (1.7-t / 2 f, 1.3)}\right) & t \geq 0\end{cases}
$$

where $C_{0}$ is a normalization constant, as shown in Figure 4. The rate is higher than one might expect from sky glow along, but the counter includes many stars within the field-of-view providing direct light into the counter.
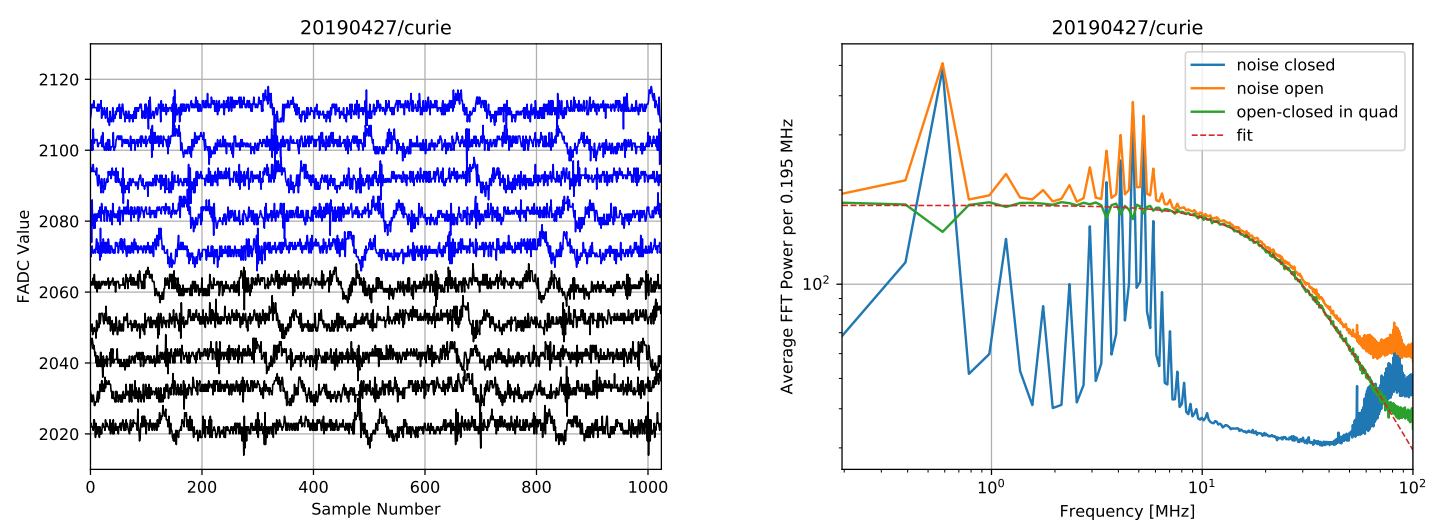

Figure 3: Left: Sample noise waveforms, shutter closed (blue) and shutter open (black). Successive waveforms have been shifted up or down by 10 FADC counts for clarity. Each Sample number corresponds to 5 ns so the entire waveform corresponds to $5.12 \mu \mathrm{s}$. Right: The average FFT power vs frequency for noise closed, noise open and their difference in quadrature. The noise closed and noise open FFTs are the average of the magnitude of the real FFT values over 4096 separate waveforms.

\section{Coincident Events between NICHE and TALE}

We searched for coincident events between NICHE and the TALE FD telescopes. We found unambiguous coincidences within 10-20 $\mu$ s. Approximately $8 \%$ of all NICHE plane-fit events are in coincidence with TALE; these events predominantly have plane normals directed towards the northwest and with large zenith angles. An example NICHE-TALE coincident event is shown in Figure 5. The TALE-Cherenkov profile constrained geometry fit (PCGF) gives quite precise determination of the shower geometry, especially for the angle of the shower axis within the showerdetector plane [6]. For the coincident events, NICHE measures the same shower axis direction within $1^{\circ}$ (the level of agreement in direction is dominated by the uncertainty in the NICHE determination of the Cherenkov plane normal). We can use this information from TALE to examine the CWLD from NICHE events, even in cases where the shower core is outside the NICHE array. 


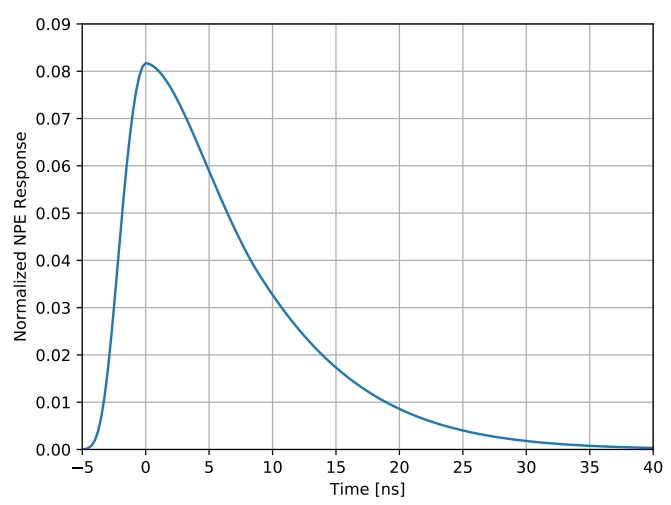

Figure 4: The impulse response function [5] that reproduces the noise FFT in Figure 3. The risetime parameter is $2.5 \mathrm{~ns}$, while the falltime parameter is $10.7 \mathrm{~ns}$. The sum of the risetime and falltime in this parameterization is very close to the FWHM.

However, the exact location of the shower core is uncertain, despite the very accurate determination of the shower angle, due to the fact that the TALE-Cherenkov data constrains the shower geometry at typically $45^{\circ}$ above the horizon and several kilometers above the ground. Some of this uncertainty is indicated in the event displayed in Figure 5. The typical uncertainty of the location of the shower core on the ground from TALE is $50-100 \mathrm{~m}$.

One can perform a hybrid timing fit between the TALE-Cherenkov data and the NICHE counter data. In this fit, the locations of the NICHE counters are projected onto the shower detector plane from the TALE event to give and angle in the SDP, and the NICHE times are adjusted to account for the speed-of-light travel time from the actual NICHE counter location to the plane, perpendicular to the shower axis at the TALE location (essentially the time to get the NICHE counter signal to TALE). There will be an overall time adjustment necessary to take into account latency times in both DAQ systems and the different transit times of the PMTs in each system. However, only one such time, which we call the ad hoc time, is needed for all the different NICHE counters in an event. With the added information and the much longer lever arm in angle, one can perform very precise geometry fits to the Time-vs-Angle data. An example fit (for the same event as above) is shown in Figure 6. In this fit, the ad hoc time is allowed to vary, which allows the NICHE times to move up and down, as a unit in the plot. However, the slope of the NICHE times with angle will not change. For all the NICHE-TALE for which we can do this fit (requiring 4 or more coincident NICHE counters in the NICHE event), we get the distribution of ad hoc times shown in Figure 6. It is remarkable the mean ad hoc time shift required is only $88 \mathrm{~ns}$ with a spread of $45 \mathrm{~ns}$. This shift is smaller than one FADC sample window at TALE. With the ad hoc shift determined one can perform NICHE-TALE hybrid fits even in the case where only a single NICHE counter is in coincidence with TALE. 

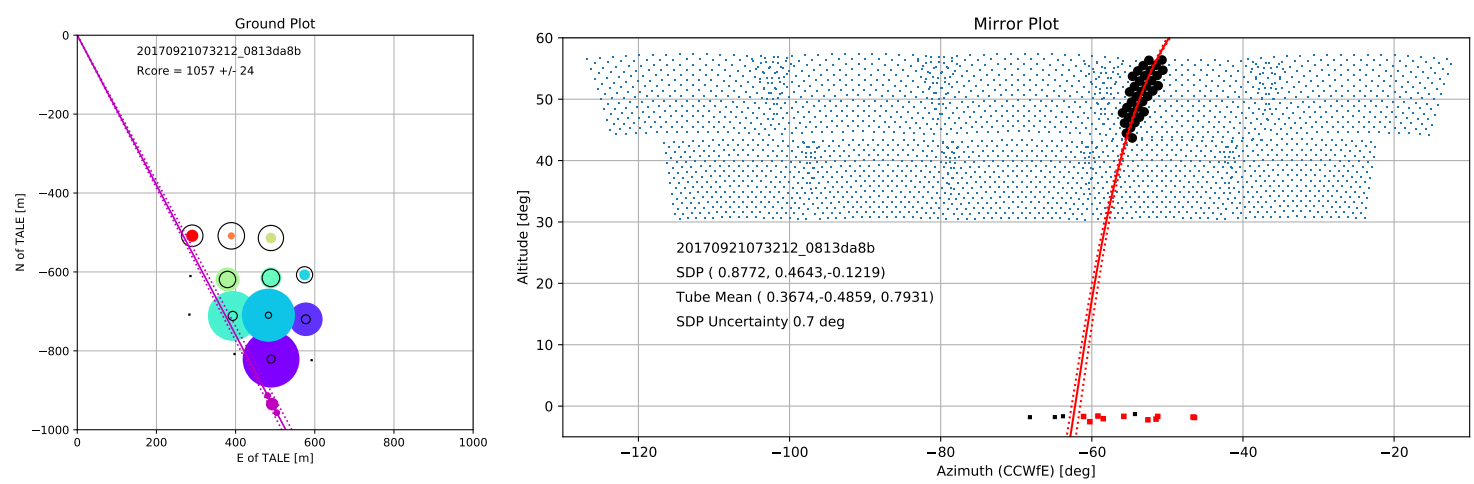

Figure 5: Left: A plot of the NICHE response to an event from 20170921. The filled color circles are drawn with centers at the locations of the NICHE counters with respect to TALE and with a radius proportional to the total signal size seen in that counter. The color of the filled circle represents the relative time of the counter trigger within the multiple, with purple being early and red being late. The size of the open black circles represents the FWHM of the signal seen by the NICHE counter. The purple line represents the location of the Shower-Detector Plane seen by TALE as projected onto the ground and with its uncertainty given by the dotted lines. Right: A plot of the triggered tubes in the TALE detector. The filled black circles represent the good, triggered PMTs with a location representing the direction viewed by the PMT and with a radius proportional to the signal size. The black dots represent the viewing directions of all the rest of the PMTS. The solid red line represents the Shower-Detector Plane as projected onto the celestial sphere, with uncertainy given by the dotted lines. The directions to the triggered NICHE counters are given by the red squares.
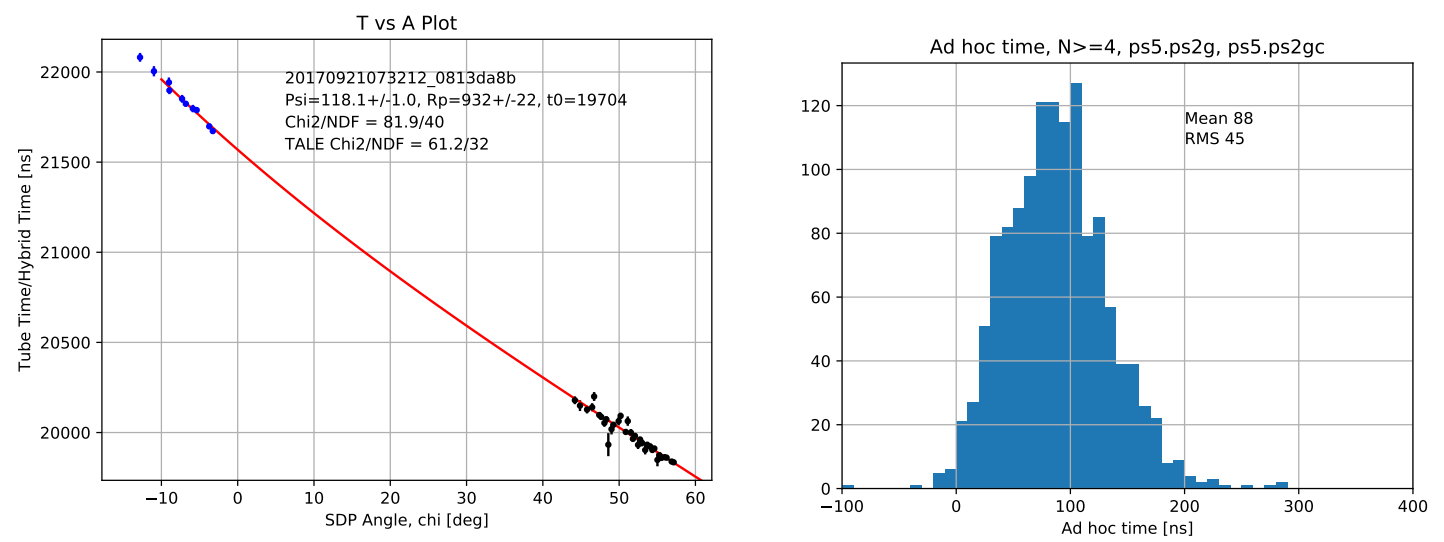

Figure 6: Left: A plot of the time vs. angle-in-the-SDP of TALE PMTs as black points with error bars. The times of NICHE triggers as projected onto the TALE position are shown as blue points. There is one ad hoc time added to all the NICHE times to get the best timing fit. The timing fit is shown as a red line. Right: The distribution of ad hoc times over all NICHE-TALE coincident events. The distribution has a mean of $88 \mathrm{~ns}$ and an RMS width of $45 \mathrm{~ns}$. 


\section{Measurement of the Dependence of the Cherenkov Width Lateral Distribution with Shower Depth}

Using the coincident NICHE-TALE events, one can measure the CWLD and its dependence on $X_{\max }$ or rather its dependence on the distance from the ground (or the counter) to the location of shower max, $D_{\max }$, using only data, without any dependence on modeling. Using the shower geometry determined by TALE, we calculate the shower core location, and the distances of each NICHE counter to the shower axis, $R_{P}$. We also use the TALE determination of $X_{\max }$ to calculate $D_{\max }$ for each counter. Each NICHE counter signal waveform is fit to the expected response function [5] to determine the FWHM of the signal. We can then plot the FWHM of the signal of each counter against its distance from the shower core. This is shown in Figure 7. In addition we color code the point according to $D_{\max }$, where observed values of $D_{\max }$ range from $0-15 \mathrm{~km}$, with the bulk being from 3-7 km. To highlight the strucure in this plot, we find the mean width for all events in 100-m bins of $R_{P}$ and 2-km bins of $D_{\max }$. Only bins with 40 or more counters contributing are plotted. The plot of $<\log _{10}(\mathrm{FWHM} / \mathrm{ns})>$ vs $R_{P}$ vs $D_{\max }$ is shown on the right in Figure 7 . There is a clear separation in the measured CWLD curves with $D_{\max }$. Recall that the individual $R_{P}$ values are uncertain at the level of $100 \mathrm{~m}$, so that there is a good bit of horizontal smearing in the plots in Figure 7.
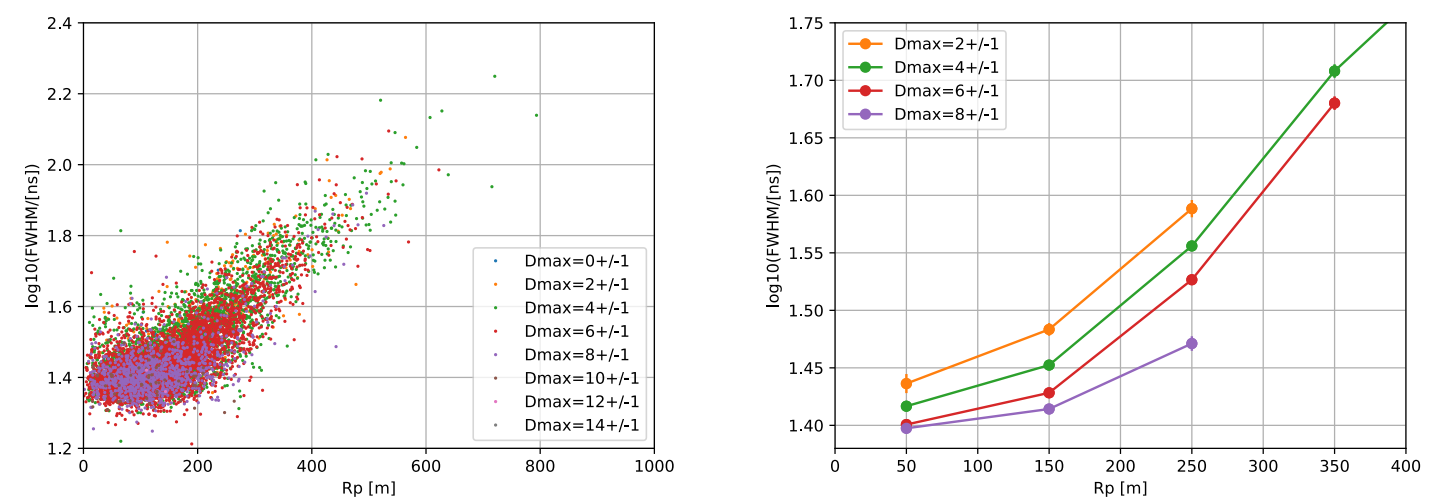

Figure 7: Left: The FWHM of NICHE signals vs the distance, $R_{P}$, from the NICHE counter to the shower axis as determined by TALE. The points are color coded by the distance $D_{\max }$ from the NICHE counter to the location of the shower maximum as determined by TALE. $D_{\max }$ range from $0-14 \mathrm{~km}$. Right: The same as in the left plot with the mean of the FWHM for a given 100-m band of $R_{P}$ and $2 \mathrm{~km}$ bank of $D_{\max }$ Only bins with more than 40 events are shown. There is a clear dependance of the FWHM with $D_{\max }$ at a given $R_{P}$.

\section{Acknowledgements}

The Telescope Array experiment is supported by the Japan Society for the Promotion of Science(JSPS) through Grants-in-Aid for Priority Area 431, for Specially Promoted Research JP21000002, for Scientific Research (S) JP19104006, for Specially Promoted Research JP15H05693, 
for Scientific Research (S) JP15H05741, for Science Research (A) JP18H03705 and for Young Scientists (A) JPH26707011; by the joint research program of the Institute for Cosmic Ray Research (ICRR), The University of Tokyo; by the U.S. National Science Foundation awards PHY-0601915, PHY-1404495, PHY-1404502, and PHY-1607727; by the National Research Foundation of Korea (2016R1A2B4014967, 2016R1A5A1013277, 2017K1A4A3015188, 2017R1A2A1A05071429) ; by the Russian Academy of Sciences, RFBR grant 16-02-00962a (INR), IISN project No. 4.4502.13, and Belgian Science Policy under IUAP VII/37 (ULB). The foundations of Dr. Ezekiel R. and Edna Wattis Dumke, Willard L. Eccles, and George S. and Dolores Doré Eccles all helped with generous donations. The State of Utah supported the project through its Economic Development Board, and the University of Utah through the Office of the Vice President for Research. The experimental site became available through the cooperation of the Utah School and Institutional Trust Lands Administration (SITLA), U.S. Bureau of Land Management (BLM), and the U.S. Air Force. We appreciate the assistance of the State of Utah and Fillmore offices of the BLM in crafting the Plan of Development for the site. Patrick Shea assisted the collaboration with valuable advice on a variety of topics. The people and the officials of Millard County, Utah have been a source of steadfast and warm support for our work which we greatly appreciate. We are indebted to the Millard County Road Department for their efforts to maintain and clear the roads which get us to our sites. We gratefully acknowledge the contribution from the technical staffs of our home institutions. An allocation of computer time from the Center for High Performance Computing at the University of Utah is gratefully acknowledged.

\section{References}

[1] B. Peters, Primary energy spectrum and air showers, Proceedings of the 6th ICRC (Moscow) 3 (1960) 157.

[2] Y. Tsunesada et al., NICHE: Non-Imaging Cherenkov Light Observation at the TA Site, JPS Conf. Proc. 19 (2018) 011042.

[3] D.R. Bergman et al., j-NICHE: Prototype detectors of a non-imaging Cherenkov array at the Telescope Array site, Proceedings of the 35th ICRC (Busan), PoS(ICRC2017)415.

[4] Y. Omura et al., NICHE detector and operations, Proceedings of the 36th ICRC (Madison).

[5] E.E. Korosteleval, L.A. Kuzmichev, V.V. Prosin, A.V. Zablotsky, The New Method of EAS Parameters Reconstruction Using the FWHM of Cherenkov Light Pulses, Proceedings of the 31st ICRC (Łódź), 0492.

[6] R.U. Abbasi et al., The Cosmic Ray Energy Spectrum between 2 PeV and 2 EeV Observed with the TALE Detector in Monocular Mode, Astrophys. J. 865 (2018) 74. [arXiv:1809.03738] 